\title{
Screening of Pea (Pisum sativum L.) Germplasm for Growth, Yield and Resistance Against Powdery Mildew under Mid-hill Conditions of Himachal Pradesh
}

\author{
Rakesh Kumar*, Manish Kumar, Sandeep Kumar and Anil Kumar
}

Dept. of Vegetable Science, Dr YS Parmar University of Horticulture and Forestry, Solan, Nauni, Himachal Pradesh (173 230), India

\section{Article History}

Manuscript No. AR1286

Received in $10^{\text {th }}$ February, 2015

Received in revised form $20^{\text {th }}$ January, 2016

Accepted in final form $4^{\text {th }}$ February, 2016

\section{Correspondence to}

*E-mail: rakeshuhfsolan@gmail.com

\section{Keywords}

Germplasm, pea, Pisum sativum L., powdery mildew, resistance

\begin{abstract}
Fifty four genotypes of pea including four check cultivars were evaluated for various important horticultural traits in a RCBD with three replications during Rabi, 201011. The observations were recorded on days to first flower (number), node at which the first flower appear (number), number of pods plant ${ }^{-1}$, pod length $(\mathrm{cm})$, number of seeds pod $^{-1}$, shelling $\%$, days to marketable maturity (number), pod yield $\left(\mathrm{kg} \mathrm{plot}^{-1}\right.$ ), pod yield ( $\left.\mathrm{q} \mathrm{ha}^{-1}\right)$, TSS $\left({ }^{\circ} \mathrm{B}\right)$, total phenols $\left(\mathrm{g} 100 \mathrm{~g}^{-1}\right)$ and powdery mildew severity (\%). Analysis of variance showed significant differences among all the genotypes for all the characters under study. In this study 4 genotypes were found earliest among all. Three genotypes were found superior to yield and yield contributing traits when compared with the performance of the check cultivars. While 23 genotypes performed better than the check cultivars among quality traits viz., total sugars (\%), total soluble solids $\left({ }^{\circ} \mathrm{B}\right)$ and phenol content $\left(\mathrm{g} 100 \mathrm{~g}^{-1}\right)$. Similarly, 18 genotypes had shown a resistant reaction to powdery mildew severity (\%). It is concluded that genotypes 10/PMVAR-3, 10/PMVAR-4, 10/PMVAR-5, 9/PMVAR-7, AG-12, IC-381058 and VP-433 were superior over other entries and over standard checks for yield, quality and other important horticultural traits. 10/PMVAR-5 was found superior to checks in most of characters like pod yield $\left(\mathrm{q} \mathrm{ha}^{-1}\right)$, number of pods plant $\mathrm{t}^{-1}$, shelling $\%$, total sugars $(\%)$ and total soluble solids $\left({ }^{\circ} \mathrm{B}\right)$. Thus it is recommended for further multilocation testing in the different regions of state.
\end{abstract}

\section{Introduction}

Pea (Pisum sativum L.) is a self pollinated annual herb, bushy or climbing plant belongs to Fabaceae family, which requires a relatively cool and humid climate. It is one of the most important vegetable crops grown extensively throughout the world during cool season. It is a rich source of proteins, carbohydrates, vitamins and crude fibers. In India, it is grown as winter season vegetable in the Central and Northern plains and as a summer and autumn-winter crop in the hilly regions. It occupies an area of about 433.6 thousand ha with annual production of 3868.6 thousand mt (Anonymous, 2014).

In Himachal Pradesh, Pea holds foremost position as leading off-season vegetable grown over an area of 23.9 thousand ha with an annual production of 271.1 thousand mt (Anonymous, 2014). It is of greater economic importance in high hills of Himachal Pradesh as produce from the hilly regions is available in plains at a time when it cannot be grown in such areas because of high temperature. Further, hilly grown pea due to its characteristic flavor, sweetness, freshness and good quality pods get a special preference from consumers in the market, thus bringing lucrative returns to growers. Thus, this crop has promising future in Himachal Pradesh.

Inspite of such an economic importance, production unit ${ }^{-1}$ area of pea is still low in the country especially when varieties are grown during off-season and the major constraints attributed to this are lack of high yielding varieties with poor or no resistance to powdery mildew. There is an urgent need to evaluate large number of genotypes and to identify high yielding and superior varieties of pea which can be released as such for commercial production or can be incorporated in the future crop improvement programmes for improvement of yield and quality traits. The present investigation was designed for a comparative study of local as well as exotic genotypes of peas under mid hill zone of Himachal Pradesh.

\section{Materials and Methods}

The investigation was carried out at Dr. Y. S. Parmar University of Horticulture and Forestry, Nauni, Solan, Himachal Pradesh, India consisting of fifty four genotypes of pea. The 
genotypes along with their sources of availability have been presented in Table 1. Seeds were directly sown in the field in the month of November, 2010 at a spacing of $(40 \times 10)$ $\mathrm{cm}$ in a plot of $(2 \times 2) \mathrm{m}^{2}$ size. There were five rows in each plot. Each genotype was sown in a Randomized Complete Block Design (RCBD) with three replications. The standard cultural practices recommended in the Package of Practices for Vegetable Crops, were followed to ensure a healthy crop stand. Agro-meteorological data during the growing season from November, 2010 to April, 2011 is presented in Table 2. The observations were recorded on 10 randomly selected plants from each replication on days to first flower (number), node at which the first flower appear (number), number of pods plant ${ }^{-1}$, pod length $(\mathrm{cm})$, number of seeds pod ${ }^{-1}$, days to marketable maturity (number), pod yield $\left(\mathrm{kg} \mathrm{plot}^{-1}\right)$, pod yield $\left(\mathrm{q} \mathrm{ha}^{-1}\right)$, TSS

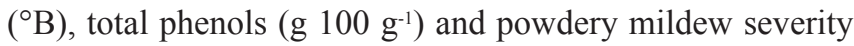
(\%) except shelling \% for which observations were recorded on 25 randomly taken pods in each genotype per replication at the time of $2^{\text {nd }}$ harvest.

Disease severity in different lines was quantified by using the disease severity scale described by Ghufranulhaq et al. (2000) is given below (Table 3).

The percent disease index (PDI) was calculated by following the formula given by McKinney (1923).

$$
\mathrm{PDI}=\frac{\text { Sum of all grades }}{\begin{array}{c}
\text { Total number of leaves assessed } \times \\
\text { maximum disease grade }
\end{array}} \times 100
$$

The statistical analysis was carried out for each observed character under the study using MS-Excel, SPSS 16.0 and SPAR 2.0 packages. The mean values of data were subjected to analysis of variance and ANOVA was set as per Gomez and Gomez (1983) for Randomized Block Design.

\section{Results and Discussion}

The analysis of variance indicated highly significant differences among the genotypes for all the traits studied. The mean performance of all the genotypes for various traits under study is given below.

\subsection{Earliness}

The minimum days to first flower were recorded in genotype 9/PMVAR-7 (75.33) which were statistically at par with 8/ PMVAR-5, 10/PMVAR-4 and 10/PMVAR-3 (Table 4). The maximum days to first flower were recorded in 9/PMVAR-5 (98.33). The performance of the check cultivars was recorded as Arka Ajit (86.33), Azad P-1 (93.00), Lincoln (89.67) and $\mathrm{Pb} .89$ (90.33). The data recorded on node at which first flower appear (number), revealed that genotype 9/PMVAR-7 (8.13) recorded lowest node at which the first flower appear which
Table 1: List of pea genotypes studied along with their

\begin{tabular}{|c|c|}
\hline Genotype & Source \\
\hline 10/PMVAR-1 & IIVR, Varanasi \\
\hline 10/PMVAR-2 & IIVR, Varanasi \\
\hline 10/PMVAR-3 & IIVR, Varanasi \\
\hline 10/PMVAR-4 & IIVR, Varanasi \\
\hline 10/PMVAR-5 & IIVR, Varanasi \\
\hline 8/PMVAR-1 & IIVR, Varanasi \\
\hline 8/PMVAR-3 & IIVR, Varanasi \\
\hline 8/PMVAR-4 & IIVR, Varanasi \\
\hline 8/PMVAR-5 & IIVR, Varanasi \\
\hline 9/PMVAR-1 & IIVR, Varanasi \\
\hline 9/PMVAR-2 & IIVR, Varanasi \\
\hline 9/PMVAR-5 & IIVR, Varanasi \\
\hline 9/PMVAR-6 & IIVR, Varanasi \\
\hline 9/PMVAR-7 & IIVR, Varanasi \\
\hline 9/PMVAR-8 & IIVR, Varanasi \\
\hline AG-12 & UHF, Solan \\
\hline AG-13 & UHF, Solan \\
\hline AG-38 & UHF, Solan \\
\hline AG-45 & UHF, Solan \\
\hline AG-55 & UHF, Solan \\
\hline AG-56 & UHF, Solan \\
\hline AG-8 & UHF, Solan \\
\hline AG-9 & UHF, Solan \\
\hline HFP-4 & NBPGR Regional Research Station, Phagli \\
\hline IC-208385 & NBPGR Regional Research Station, Phagli \\
\hline IC-208387 & NBPGR Regional Research Station, Phagli \\
\hline IC-209114 & NBPGR Regional Research Station, Phagli \\
\hline IC-218982 & NBPGR Regional Research Station, Phagli \\
\hline IC-218985 & NBPGR Regional Research Station, Phagli \\
\hline IC-218999 & NBPGR Regional Research Station, Phagli \\
\hline IC-267138 & NBPGR Regional Research Station, Phagli \\
\hline IC-26752 & NBPGR Regional Research Station, Phagli \\
\hline IC-280357 & NBPGR Regional Research Station, Phagli \\
\hline IC-291541 & NBPGR Regional Research Station, Phagli \\
\hline IC-296678 & NBPGR Regional Research Station, Phagli \\
\hline IC-310833 & NBPGR Regional Research Station, Phagli \\
\hline IC-311065 & NBPGR Regional Research Station, Phagli \\
\hline IC-311067 & NBPGR Regional Research Station, Phagli \\
\hline IC-311069 & NBPGR Regional Research Station, Phagli \\
\hline IC-311070 & NBPGR Regional Research Station, Phagli \\
\hline IC-342025 & NBPGR Regional Research Station, Phagli \\
\hline
\end{tabular}
sources of availability 


\begin{tabular}{ll}
\hline Genotype & Source \\
\hline IC-381058 & NBPGR Regional Research Station, Phagli \\
IP-3 & IIVR, Varanasi \\
KS-205 & IIVR, Varanasi \\
KS-210 & IIVR, Varanasi \\
RACHNA & NBPGR Regional Research Station, Phagli \\
VL-8 & NBPGR Regional Research Station, Phagli \\
VP-433 & NBPGR Regional Research Station, Phagli \\
VP434 & NBPGR Regional Research Station, Phagli \\
CHP-2 & NBPGR Regional Research Station, Phagli \\
${ }^{*}$ ARKA AJIT & NBPGR Regional Research Station, Phagli \\
${ }^{*}$ AZAD P-1 & NBPGR Regional Research Station, Phagli \\
${ }^{*}$ LINCOLN & NBPGR Regional Research Station, Phagli \\
*PB.89 & NBPGR Regional Research Station, Phagli \\
\hline
\end{tabular}

${ }^{*}$ Check cultivars (s)

Table 2: Agro-meteorological data during the growing season from November, 2010 to April, 2011

\begin{tabular}{|c|c|c|c|c|c|}
\hline \multirow[t]{2}{*}{ Month } & \multicolumn{3}{|c|}{ Temperature $\left({ }^{\circ} \mathrm{C}\right)$} & \multirow{2}{*}{$\begin{array}{c}\text { Relative } \\
\text { Humidity } \\
(\%)\end{array}$} & \multirow{2}{*}{$\begin{array}{c}\text { Rainfall } \\
(\mathrm{mm})\end{array}$} \\
\hline & $\begin{array}{l}\text { Maxi- } \\
\text { mum }\end{array}$ & $\begin{array}{l}\text { Mini- } \\
\text { mum }\end{array}$ & Mean & & \\
\hline November & 23.59 & 5.93 & 14.76 & 21.80 & 53.42 \\
\hline December & 19.89 & 1.20 & 10.55 & 70.20 & 57.82 \\
\hline January & 18.11 & 0.42 & 9.27 & 23.20 & 53.66 \\
\hline February & 17.18 & 0.25 & 8.72 & 61.50 & 54.18 \\
\hline March & 24.38 & 8.34 & 16.36 & 18.20 & 48.39 \\
\hline April & 25.63 & 10.33 & 17.98 & 33.70 & 48.98 \\
\hline
\end{tabular}

Table 3: Disease severity scale

\begin{tabular}{lcl}
\hline Disease grade & $\begin{array}{c}\text { Percent leaf area } \\
\text { infected }\end{array}$ & Disease reaction \\
\hline 0 & No infection & Immune \\
1 & $0-10$ & Resistant \\
2 & $10.1-30$ & Moderately resistant \\
3 & $30.1-70$ & Moderately susceptible \\
4 & $70.1-80$ & Susceptible \\
5 & $80.1-100$ & Highly Susceptible \\
\hline
\end{tabular}

was statistically at par with two other genotypes viz., 10/ PMVAR-4 (8.93) and 8/PMVAR-5 (8.33). However, highest node at which first flower appear was recorded in 9/PMVAR-5 (17.33). Whereas, the check varieties recorded as Arka Ajit (12.53), Azad P-1 (15.20), Lincoln (13.87), Pb.89 (14.13) node at which the first flower appear. The mean values for different genotypes revealed that 9/PMVAR-7 (137.33) was the earliest for attaining marketable maturity which was statistically at par with 10/PMVAR-3 (141.33), 10/PMVAR-4 (139.33) and 8/PMVAR-5 (138.00). The genotype 9/PMVAR-5 (160.33) took maximum days to attain marketable maturity. Check varieties Arka Ajit, Azad P-1, Lincoln and Pb.89 needed $148.33,155.00,151.67$ and 152.33 days respectively to attain marketable maturity.

\subsection{Yield and component traits}

A comparison of mean values of the genotypes for number of pods plant ${ }^{-1}$ (Table 3 ) depicted maximum number of pods plant $^{-1}$ in 10/PMVAR-5 (38.35) which was statistically at par with 10/PMVAR-4 (36.64). Minimum number of pods plant ${ }^{-1}$ were recorded in AG-12 (17.65). However check varieties Arka Ajit, Azad P-1, Lincoln and Pb.89 gave 27.73, 34.57, 32.59 and 33.31 pods plant $^{-1}$, respectively. Maximum pod length was recorded in VP-433 (8.47) and it was found statistically at par with 7 genotypes including three checks viz., 10/PMVAR-5 (8.00), KS-205 (8.13), KS-210 (7.80), CHP-2 (7.80), Azad P-1 (7.80), Lincoln (7.70) and Pb.89 (7.80). Minimum pod length was observed in AG-8 (4.83). Number of seeds pod ${ }^{-1}$ indicates that maximum number of seeds pod $^{-1}$ were recorded in VP-433 (8.30) and it was statistically at par with 9 genotypes including all checks viz., 10/PMVAR-5 (7.84), KS-205 (7.97), KS-210 (7.64), VL-8 (7.32), CHP-2 (7.64), Arka Ajit (7.35), Azad P-1 (7.64), Lincoln (7.55) and Pb.89 (7.64) whereas AG-8 (4.74) recorded minimum number of seeds pod $^{-1}$.

Maximum shelling \% was observed in VP-433 (73.27) which was statistically at par with 10/PMVAR-5 (68.24) and KS-205 (69.68). However, minimum shelling \% was recorded in AG-8 (34.10). The check varieties Arka Ajit, Azad P-1, Lincoln and $\mathrm{Pb} .89$ gave $62.85 \%, 66.08 \%, 65.01 \%$ and $66.08 \%$ shelling $\%$ respectively. AG-12 with $140.29 \mathrm{~cm}$ recorded maximum plant height which was statistically at par with 24 genotypes. Whereas, 10/PMVAR-4 recorded minimum plant height i.e., $81.42 \mathrm{~cm}$. The performance of check varieties to plant height was viz., Arka Ajit (109.04), Azad P-1 (87.83), Lincoln (93.97) and $\mathrm{Pb} .89$ (91.74 cm). Maximum pod yield plot ${ }^{-1}$ was observed in 10/PMVAR-5 $(6.16 \mathrm{~kg})$ which was statistically at par with check namely Azad P-1 (5.38) whereas, minimum pod yield plot $^{-1}$ was recorded in AG-12 $(1.63 \mathrm{~kg})$. Other three check varieties Arka Ajit, Lincoln and Pb.89 recorded 4.13, 4.96 and $5.17 \mathrm{~kg}$ pod yield plot $^{-1}$, respectively. Maximum pod yield hectare $^{-1}$ was observed in 10/PMVAR-5 (138.63 q) which was statistically at par with check Azad P-1 (120.96 q). Minimum pod yield ha ${ }^{-1}$ was recorded in AG-12 (36.70 q). Other three check varieties Arka Ajit, Lincoln and Pb.89 recorded 92.87, 111.53 and 116.22 q pod yield ha ${ }^{-1}$, respectively.

\subsection{Quality traits}

The mean values presented in Table 4 for different genotypes revealed that maximum total soluble solids were recorded 


\begin{tabular}{|c|c|c|c|c|c|c|c|c|c|c|c|c|c|c|}
\hline $\begin{array}{l}\text { Geno- } \\
\text { type }\end{array}$ & DFF & NFF & NPP & PL & NSP & SP & $\mathrm{PH}$ & DMM & PYPP & PYPH & PMS & TS & $\mathrm{TP}$ & TSS \\
\hline $\begin{array}{l}\text { 10/PM- } \\
\text { VAR-1 }\end{array}$ & 92.33 & 14.93 & 23.50 & 7.17 & 7.02 & 59.26 & 122.15 & 154.33 & 3.30 & 74.24 & 10.54 & $5.02(2.24)$ & 0.42 & $61.26(51.50)$ \\
\hline $\begin{array}{l}\text { 10/PM- } \\
\text { VAR-2 }\end{array}$ & 88.00 & 13.20 & 24.40 & 6.73 & 6.60 & 54.59 & 119.36 & 150.00 & 3.08 & 69.39 & 9.78 & $4.69(2.16)$ & 0.38 & $64.72(53.60)$ \\
\hline $\begin{array}{l}\text { 10/PM- } \\
\text { VAR-3 }\end{array}$ & 79.33 & 9.87 & 32.68 & 7.17 & 7.02 & 59.26 & 93.69 & 141.33 & 4.42 & 99.47 & 14.46 & $6.72(2.58)$ & 0.58 & $43.24(41.00)$ \\
\hline $\begin{array}{l}\text { 10/PM- } \\
\text { VAR-4 }\end{array}$ & 77.33 & 8.93 & 36.64 & 6.50 & 6.37 & 52.07 & 81.42 & 139.33 & 4.19 & 94.27 & 13.65 & $6.37(2.49)$ & 0.55 & $46.95(43.02)$ \\
\hline $\begin{array}{l}\text { 10/PM- } \\
\text { VAR-5 }\end{array}$ & 86.00 & 12.53 & 38.35 & 8.00 & 7.84 & 68.24 & 85.94 & 148.00 & 6.16 & 138.63 & 20.54 & $9.37(3.05)$ & 0.85 & $15.26(21.89)$ \\
\hline $\begin{array}{l}\text { 8/PM- } \\
\text { VAR-1 }\end{array}$ & 91.00 & 14.40 & 23.77 & 6.83 & 6.70 & 55.66 & 121.31 & 153.00 & 3.08 & 69.27 & 9.77 & $4.68(2.16)$ & 0.38 & $64.81(53.61)$ \\
\hline $\begin{array}{l}\text { 8/PM- } \\
\text { VAR-3 }\end{array}$ & 94.00 & 15.20 & 25.57 & 6.83 & 6.70 & 55.66 & 115.73 & 156.00 & 3.30 & 74.15 & 10.52 & $5.01(2.23)$ & 0.42 & $61.32(51.62)$ \\
\hline $\begin{array}{l}\text { 8/PM- } \\
\text { VAR-4 }\end{array}$ & 84.33 & 11.73 & 22.96 & 6.40 & 6.27 & 50.99 & 123.82 & 146.33 & 2.69 & 60.63 & 8.42 & $4.10(2.02)$ & 0.33 & $70.98(57.40)$ \\
\hline $\begin{array}{l}\text { 8/PM- } \\
\text { VAR-5 }\end{array}$ & 76.00 & 8.33 & 28.45 & 6.83 & 6.70 & 55.66 & 106.81 & 138.00 & 3.59 & 80.78 & 11.55 & $5.46(2.33)$ & 0.46 & $56.58(48.79)$ \\
\hline $\begin{array}{l}\text { 9/PM- } \\
\text { VAR-1 }\end{array}$ & 91.33 & 14.53 & 27.10 & 6.83 & 6.70 & 55.66 & 110.99 & 153.33 & 3.44 & 77.47 & 11.04 & $5.23(2.28)$ & 0.44 & $58.95(50.19)$ \\
\hline $\begin{array}{l}\text { 9/PM- } \\
\text { VAR-2 }\end{array}$ & 94.33 & 15.73 & 21.16 & 6.40 & 6.27 & 50.99 & 129.40 & 156.33 & 2.53 & 56.82 & 7.83 & 3.84 (1.96) & 0.30 & $73.70(59.14)$ \\
\hline $\begin{array}{l}\text { 9/PM- } \\
\text { VAR-5 }\end{array}$ & 98.33 & 17.33 & 25.30 & 6.83 & 6.70 & 55.66 & 116.57 & 160.33 & 3.26 & 73.25 & 10.38 & $4.95(2.22)$ & 0.41 & $61.96(51.95)$ \\
\hline $\begin{array}{l}\text { 9/PM- } \\
\text { VAR-6 }\end{array}$ & 95.67 & 16.27 & 28.72 & 6.50 & 6.37 & 52.07 & 105.97 & 157.67 & 3.31 & 74.49 & 10.58 & $5.03(2.24)$ & 0.42 & $61.08(51.39)$ \\
\hline $\begin{array}{l}\text { 9/PM- } \\
\text { VAR-7 }\end{array}$ & 75.33 & 8.13 & 34.48 & 6.40 & 6.27 & 50.99 & 88.11 & 137.33 & 3.77 & 84.80 & 12.18 & $5.73(2.39)$ & 0.49 & $53.71(47.11)$ \\
\hline $\begin{array}{l}\text { 9/PM- } \\
\text { VAR-8 }\end{array}$ & 87.33 & 12.93 & 24.31 & 7.17 & 7.02 & 59.26 & 119.64 & 149.33 & 3.42 & 76.86 & 10.94 & $5.19(2.27)$ & 0.43 & $59.39(50.47)$ \\
\hline AG-12 & 94.00 & 15.60 & 17.65 & 5.17 & 5.06 & 37.70 & 140.29 & 156.00 & 1.63 & 36.70 & 4.70 & $2.48(1.57)$ & 0.89 & $10.73(19.10)$ \\
\hline AG-13 & 89.67 & 13.87 & 19.00 & 6.07 & 5.95 & 47.40 & 136.10 & 151.67 & 2.15 & 48.29 & 6.51 & $3.26(1.80)$ & 0.90 & $9.14(17.58)$ \\
\hline AG-38 & 92.67 & 15.07 & 22.60 & 5.17 & 5.06 & 37.70 & 124.94 & 154.67 & 1.94 & 43.72 & 5.79 & $2.95(1.70)$ & 0.90 & $9.76(18.18)$ \\
\hline AG-45 & 84.67 & 11.87 & 18.28 & 5.17 & 5.06 & 37.70 & 138.33 & 146.67 & 1.67 & 37.56 & 4.84 & 2.54 (1.59) & 0.89 & 10.61 (18.99) \\
\hline AG-55 & 87.00 & 12.80 & 20.71 & 5.40 & 5.29 & 40.21 & 130.80 & 149.00 & 1.93 & 43.32 & 5.73 & $2.93(1.71)$ & 0.90 & $9.82(18.25)$ \\
\hline AG-56 & 84.33 & 11.73 & 20.35 & 5.83 & 5.72 & 44.88 & 131.92 & 146.33 & 2.15 & 48.33 & 6.51 & $3.27(1.80)$ & 0.90 & $9.13(17.56)$ \\
\hline AG-8 & 91.00 & 14.40 & 20.35 & 4.83 & 4.74 & 34.10 & 131.92 & 153.00 & 1.63 & 36.75 & 4.71 & $2.48(1.57)$ & 0.89 & $10.72(19.10)$ \\
\hline AG-9 & 90.33 & 14.13 & 19.54 & 5.40 & 5.29 & 40.21 & 134.43 & 152.33 & 1.85 & 41.59 & 5.46 & $2.81(1.67)$ & 0.89 & $10.06(18.48)$ \\
\hline HFP-4 & 85.67 & 12.27 & 19.72 & 5.17 & 5.06 & 37.70 & 133.87 & 147.67 & 1.76 & 39.61 & 5.16 & $2.68(1.63)$ & 0.19 & $85.99(68.20)$ \\
\hline $\begin{array}{l}\text { IC- } \\
208385\end{array}$ & 92.67 & 15.07 & 19.27 & 7.07 & 6.93 & 58.18 & 135.26 & 154.67 & 2.76 & 62.01 & 8.64 & $4.19(2.04)$ & 0.92 & $7.26(15.59)$ \\
\hline $\begin{array}{l}\text { IC- } \\
208387\end{array}$ & 84.33 & 11.73 & 20.80 & 5.17 & 5.06 & 37.70 & 130.52 & 146.33 & 1.83 & 41.13 & 5.39 & $2.78(1.65)$ & 0.89 & $10.12(18.52)$ \\
\hline $\begin{array}{l}\text { IC- } \\
209114\end{array}$ & 85.00 & 12.00 & 21.34 & 6.50 & 6.37 & 52.07 & 128.85 & 147.00 & 2.60 & 58.61 & 8.11 & $3.96(1.99)$ & 0.92 & $7.72(16.13)$ \\
\hline
\end{tabular}




\begin{tabular}{|c|c|c|c|c|c|c|c|c|c|c|c|c|c|c|}
\hline $\begin{array}{l}\text { Geno- } \\
\text { type }\end{array}$ & DFF & NFF & NPP & PL & NSP & SP & $\mathrm{PH}$ & DMM & PYPP & PYPH & PMS & TS & $\mathrm{TP}$ & TSS \\
\hline $\begin{array}{l}\text { IC- } \\
218982\end{array}$ & 87.00 & 12.80 & 22.42 & 5.70 & 5.59 & 43.45 & 125.50 & 149.00 & 2.24 & 50.34 & 6.82 & $3.40(1.83)$ & 0.91 & $8.86(17.27)$ \\
\hline $\begin{array}{l}\text { IC- } \\
218985\end{array}$ & 81.33 & 10.53 & 20.17 & 5.47 & 5.36 & 40.93 & 132.47 & 143.33 & 1.93 & 43.45 & 5.75 & $2.94(1.71)$ & 0.90 & $9.80(18.23)$ \\
\hline $\begin{array}{l}\text { IC- } \\
218999\end{array}$ & 84.00 & 11.60 & 19.54 & 5.47 & 5.36 & 40.93 & 134.43 & 146.00 & 1.88 & 42.40 & 5.59 & $2.86(1.69)$ & 0.90 & $9.95(18.37)$ \\
\hline $\begin{array}{l}\text { IC- } \\
267138\end{array}$ & 92.00 & 14.80 & 19.81 & 5.03 & 4.93 & 36.26 & 133.59 & 154.00 & 1.70 & 38.31 & 4.95 & $2.59(1.61)$ & 0.89 & $10.51(18.90)$ \\
\hline $\begin{array}{l}\text { IC- } \\
26752\end{array}$ & 96.00 & 16.40 & 22.78 & 5.80 & 5.68 & 44.52 & 124.38 & 158.00 & 2.32 & 52.12 & 7.10 & $3.52(1.87)$ & 0.91 & $8.61(17.01)$ \\
\hline $\begin{array}{l}\text { IC- } \\
280357\end{array}$ & 85.00 & 12.00 & 18.46 & 5.80 & 5.68 & 44.52 & 137.77 & 147.00 & 1.96 & 44.11 & 5.85 & $2.98(1.72)$ & 0.90 & $9.71(18.14)$ \\
\hline $\begin{array}{l}\text { IC- } \\
291541\end{array}$ & 82.33 & 10.93 & 22.96 & 6.37 & 6.24 & 50.63 & 123.82 & 144.33 & 2.67 & 60.10 & 8.34 & $4.06(2.01)$ & 0.92 & $7.52(15.91)$ \\
\hline $\begin{array}{l}\text { IC- } \\
296678\end{array}$ & 92.67 & 15.07 & 20.17 & 5.13 & 5.03 & 37.34 & 132.47 & 154.67 & 1.77 & 39.72 & 5.17 & $2.68(1.64)$ & 0.89 & $10.31(18.72)$ \\
\hline $\begin{array}{l}\text { IC- } \\
310833\end{array}$ & 90.33 & 14.13 & 21.70 & 6.13 & 6.01 & 48.12 & 127.73 & 152.33 & 2.44 & 55.00 & 7.55 & $3.72(1.92)$ & 0.91 & $8.22(16.60)$ \\
\hline $\begin{array}{l}\text { IC- } \\
311065\end{array}$ & 95.00 & 16.00 & 22.78 & 6.03 & 5.91 & 47.04 & 124.38 & 157.00 & 2.47 & 55.47 & 7.62 & $3.75(1.92)$ & 0.91 & $8.15(16.54)$ \\
\hline $\begin{array}{l}\text { IC- } \\
311067\end{array}$ & 86.33 & 12.53 & 22.06 & 6.13 & 6.01 & 48.12 & 126.61 & 148.33 & 2.44 & 54.85 & 7.52 & $3.71(1.92)$ & 0.91 & 8.24 (16.67) \\
\hline $\begin{array}{l}\text { IC- } \\
311069\end{array}$ & 91.33 & 14.53 & 20.98 & 5.80 & 5.68 & 44.52 & 129.96 & 153.33 & 2.16 & 48.60 & 6.55 & $3.28(1.81)$ & 0.90 & $9.10(17.54)$ \\
\hline $\begin{array}{l}\text { IC- } \\
311070\end{array}$ & 81.67 & 10.67 & 21.52 & 6.37 & 6.24 & 50.63 & 128.29 & 143.67 & 2.54 & 57.13 & 7.88 & $3.86(1.96)$ & 0.91 & $7.93(16.33)$ \\
\hline $\begin{array}{l}\text { IC- } \\
342025\end{array}$ & 92.33 & 14.93 & 22.60 & 5.80 & 5.68 & 44.52 & 124.94 & 154.33 & 2.28 & 51.39 & 6.99 & $3.47(1.86)$ & 0.91 & $8.71(17.16)$ \\
\hline $\begin{array}{l}\text { IC- } \\
381058\end{array}$ & 88.33 & 13.33 & 21.70 & 7.13 & 6.99 & 58.90 & 127.73 & 150.33 & 3.08 & 69.32 & 9.77 & $4.68(2.16)$ & 0.93 & $6.26(14.40)$ \\
\hline IP-3 & 86.67 & 12.67 & 30.34 & 7.03 & 6.89 & 57.82 & 100.95 & 148.67 & 3.98 & 89.66 & 12.93 & $6.06(2.46)$ & 0.52 & $50.24(45.13)$ \\
\hline KS-205 & 84.67 & 11.87 & 23.68 & 8.13 & 7.97 & 69.68 & 121.59 & 146.67 & 4.11 & 92.40 & 13.36 & $6.24(2.50)$ & 0.54 & $48.28(44.00)$ \\
\hline KS-210 & 93.33 & 15.33 & 32.41 & 7.80 & 7.64 & 66.08 & 94.53 & 155.33 & 5.04 & 113.37 & 16.62 & $7.66(2.77)$ & 0.68 & $33.31(35.22)$ \\
\hline $\begin{array}{l}\text { RACH- } \\
\text { NA }\end{array}$ & 91.00 & 14.40 & 21.52 & 7.37 & 7.22 & 61.41 & 128.29 & 153.00 & 3.28 & 73.88 & 10.48 & $4.99(2.22)$ & 0.41 & $61.51(51.86)$ \\
\hline VL-8 & 92.00 & 14.67 & 28.72 & 7.47 & 7.32 & 62.49 & 105.97 & 154.00 & 4.20 & 94.40 & 13.67 & $6.38(2.52)$ & 0.55 & $46.86(43.18)$ \\
\hline VP-433 & 85.33 & 12.13 & 25.30 & 8.47 & 8.30 & 73.27 & 116.57 & 147.33 & 4.70 & 105.70 & 15.43 & $7.14(2.67)$ & 0.63 & $38.78(38.43)$ \\
\hline VP434 & 89.67 & 13.87 & 23.50 & 7.37 & 7.22 & 61.41 & 122.15 & 151.67 & 3.44 & 77.46 & 11.04 & $5.23(2.28)$ & 0.44 & $58.96(50.19)$ \\
\hline CHP-2 & 94.00 & 15.60 & 30.43 & 7.80 & 7.64 & 66.08 & 100.67 & 156.00 & 4.77 & 107.36 & 15.69 & 7.25 (2.69) & 0.30 & $37.60(37.76)$ \\
\hline $\begin{array}{l}\text { ARKA } \\
\text { AJIT }\end{array}$ & 86.33 & 12.53 & 27.73 & 7.50 & 7.35 & 62.85 & 109.04 & 148.33 & 4.13 & 92.87 & 13.43 & $6.28(2.50)$ & 0.89 & $7.54(14.75)$ \\
\hline $\begin{array}{l}\text { AZAD } \\
\text { P-1 }\end{array}$ & 93.00 & 15.20 & 34.57 & 7.80 & 7.64 & 66.08 & 87.83 & 155.00 & 5.38 & 120.96 & 17.80 & $8.17(2.85)$ & 0.35 & $27.89(30.66)$ \\
\hline $\begin{array}{l}\text { LIN- } \\
\text { COLN }\end{array}$ & 89.67 & 13.87 & 32.59 & 7.70 & 7.55 & 65.01 & 93.97 & 151.67 & 4.96 & 111.53 & 16.33 & $7.54(2.74)$ & 0.31 & $34.62(35.91)$ \\
\hline PB.89 & 90.33 & 14.13 & 33.31 & 7.80 & 7.64 & 66.08 & 91.74 & 152.33 & 5.17 & 116.22 & 17.06 & $7.90(2.80)$ & 0.38 & 31.27 (33.97) \\
\hline
\end{tabular}




\begin{tabular}{llllllllllllllll}
\hline $\begin{array}{l}\text { Geno- } \\
\text { type }\end{array}$ & DFF & NFF & NPP & PL & NSP & SP & PH & DMM & PYPP & PYPH & PMS & TS & TP & TSS \\
\hline Mean & 88.40 & 13.35 & 24.46 & 6.48 & 6.35 & 51.86 & 119.36 & 150.40 & 3.05 & 68.52 & 9.65 & $4.63(2.11)$ & 0.68 & $30.62(31.86)$ \\
SEm \pm & 1.77 & 0.56 & 0.72 & 0.33 & 0.38 & 4.13 & 5.45 & 2.12 & 0.29 & 6.44 & 1.19 & 0.52 & 0.03 & 4.05 \\
CD & 5.02 & 1.59 & 2.05 & 0.92 & 1.06 & 6.88 & 15.42 & 5.99 & 0.81 & 18.22 & 3.37 & 0.32 & 0.07 & 7.33
\end{tabular}

$(p=0.05)$

Where, DFF: Days to first flower; NFF: Node at which first flower appear; NPP: Number of pods plant ${ }^{-1}$, PL: Pod length; NSP: Number of seeds pod $^{-1}$, SP: Shelling \%; PH: Plant height; DMM: Days to marketable maturity; PMS: Powdery mildew severity; TS: Total sugars; TP: Total phenols; TSS: Total soluble solids; PYPP: Pod yield plot ${ }^{-1}$; PYPH: Pod yield ha ${ }^{-1}$

in 10/PMVAR-5 $\left(20.54^{\circ} \mathrm{B}\right)$ and was statistically at par with Azad P-1 $\left(17.80^{\circ} \mathrm{B}\right)$ whereas, minimum total soluble solids were observed in AG-12 $\left(4.70^{\circ} \mathrm{B}\right)$. Other three check varieties Arka Ajit, Lincoln and Pb.89 recorded 13.43, 16.33 and 17.06 ${ }^{\circ} \mathrm{B}$, respectively. Maximum total Sugars were recorded in 10/ PMVAR-5 (9.37\%) which was found statistically superior over all other genotypes. Maximum total Sugars were recorded in 10/PMVAR-5 (9.37\%). The check varieties Arka Ajit, Azad P-1, Lincoln and Pb.89 recorded 6.28, 8.17, 7.54 and $7.85 \%$ total Sugars, respectively. All the genotypes under study showed significant differences for total phenols (g 100 $\left.\mathrm{g}^{-1}\right)$. Maximum total phenols were recorded in IC-381058 $\left(0.93 \mathrm{~g} 100 \mathrm{~g} \mathrm{~g}^{-1}\right)$ and were found statistically at par with 26 other genotypes. Whereas, HFP-4 (0.19 g $\left.100 \mathrm{~g}^{-1}\right)$ recorded minimum total phenols. The check varieties Arka Ajit, Azad P-1, Lincoln and Pb.89 recorded 0.89, 0.35, 0.31 and $0.38 \mathrm{~g}$ $100 \mathrm{~g}^{-1}$ total phenols, respectively.

\subsection{Powdery mildew severity (\%)}

The data recorded on powdery mildew severity (\%) varied from IC-381058 (6.26\%) to HFP-4 (85.99\%). Twenty six genotypes were found to be at par with the most resistant genotype IC381058. Only Arka Ajit (7.17\%) had shown resistant reaction to powdery mildew disease among four checks.

Among the horticultural characters, days to first flower, node at which first flower appear and days to marketable maturity are the traits which determine the earliness of a variety. In the present study 4 genotypes were found earliest among all genotypes and can be incorporated in breeding programmes to develop early cultivars in pea. Similarly, pod yield, pod length, number of pods plant ${ }^{-1}$, number of seeds pod $^{-1}$, shelling $\%$ are the major yield and yield contributing traits. Three genotypes were found superior to yield and yield contributing traits when compared with the performance of the check cultivars in the present study and these three can be used in crop improvement programmes for improving yield. While 23 genotypes performed better than the check cultivars among quality traits viz., total sugars, total soluble solids and phenol content. These 23 genotypes can be involved in crop improvement programmes on pea to enhance quality. Similarly
18 genotypes had shown a resistant reaction to powdery mildew severity (\%). Results corroborated with the findings of Shah et al. (1990), Arshad et al. (1998); Kumar et al. (1998)); Ali et al. (2002); Mehta et al. (2005); Singh and Mir (2005); Singh et al. (2006); Bozoglu et al. (2007); Kumar et al. (2007); Sardana et al. (2007); Singh et al. (2007); Kumar (2008); Chadha et al. (2008); Nawab et al. (2008); Nishar et al. (2008); Guleria et al. (2009); Shahid et al. (2010) in pea.

\section{Conclusion}

Genotypes 10/PMVAR-3, 10/PMVAR-4, 10/PMVAR-5, 9/ PMVAR-7, AG-12, IC-381058 and VP-433 were superior over others including standard checks for yield, quality and other important horticultural traits. 10/PMVAR-5 was found superior to checks in most of characters like pod yield $\left(\mathrm{kg} \mathrm{plot}^{-1}\right)$, pod yield ( $\left.\mathrm{q} \mathrm{ha} \mathrm{h}^{-1}\right)$, number of pods plant ${ }^{-1}$, shelling percentage $(\%)$, total sugars $(\%)$ and total soluble solids $\left({ }^{\circ} \mathrm{B}\right)$. Thus it is recommended for multi-location testing in the different regions of Himachal Pradesh.

\section{References}

Ali, I., Rub, A., Hussain, S.A., 2002. Screening of pea germplasm for growth, yield and resistance against powdery mildew under the agro-climatic conditions of Peshawar. Sarhad Journal of Agriculture 18(2), 177-181.

Anonymous, 2014. Handbook of Indian Horticulture Database, NHB, Gurgaon.

Arshad, M., Hussain, S.A., Ali, S.A.N., Muhammad, N., Ziaullah, 1998. Screening of pea (Pisum sativum L.) cultivars in Kohat valley. Sarhad Journal of Agriculture 14(6), 559-562.

Bozoglu, H., Peksen, E., Peksen, A., Gulumser, A., 2007. Determination of the yield performance and harvesting periods of fifteen pea (Pisum sativum L.) cultivars sown in autumn and spring. Pakistan Journal of Botany 39(6), 2017-2025.

Chadha, S., Sharma, R., Chaudhary, D.R., Vidyasagar, 2008. Genetic variability studies in summer pea under cold desert areas of North-Western Himalayas. Agricultural Science Digest 28 (1), 10-13. 
Gomez, K.A., Gomez, A.A., 1983. Statistical Procedures for Agricultural Research. John Wiley and Sons Inc., New York, 335.

Guleria, S., Chongtham, N., Dua, S., 2009. Genetic variability, correlation and path analysis studies in pea (Pisum sativum L.). Crop Research 38(1/3), 179-183.

Kumar, B., 2008. Variability, heritability and genetic advance in pea (Pisum sativum L.). International Journal of Plant Sciences Muzaffarnagar 3(1), 211-212.

Kumar, D., Malik, B.P.S., Raj, L., 1998. Genetic variability and correlation studies in field pea (Pisum sativum L.). Legume Research 21(1), 23-29.

Kumar, R., Dhari, R., Kumar, R., Malik, B.P.S., 2007. Assessment of morphological variability and genetic diversity in pea germplasm (Pisum sativum L.). National Journal of Plant Improvement 9(1), 5-8.

Mehta, S., Kohli, U.K., Mehta, D., Kumar, D., 2005. Genetic variability studies in pea (Pisum sativum L.). Haryana Journal of Horticultural Sciences 34(1/2), 140-141.

Natarajan, S.R., Arumugam, 1983. Evaluation of pea (Pisum sativum L.) cultivars of Kodiakanal hills. South Indian Horticulture 31(1), 7-10.

Nawab, N.N., Subhani, G.M., Khalid, M., Qamar, S., Akhtar, S., 2008. Genetic variability, correlation and path analysis studies in garden pea (Pisum sativum L.). Journal of Agricultural Research Lahore. 46(4), 333-340.
Nishar, M., Ghafoor, A., Ahmad, H., Khan, M.R., Qureshi, A.S., Ali, H., Islam, M., 2008. Evaluation of genetic diversity of pea germplasm through phenotypic trait analysis. Pakistaan Journal of Botany 40(5), 2081-2086.

Sardana, S., Mahajan, R.K., Gautam, N.K., Ram, B., 2007. Genetic variability in pea (Pisum sativum $\mathrm{L}$.) germplasm for utilization. SABRAO Journal of Breeding and Genetics 39(1), 31-41.

Shah, A., Lal, S.D., Shah, A., 1990. Comparative performance of some pea cultivars under rainfed condition of U.P. hills. Progressive Horticulture. 22(1-4), 121-124.

Shahid, M., Shah, S.F.A., Ghufranulhaq, Ali, H., Ishtiaq, S., 2010. Resistance in pea germplasm lines to powdery mildew under natural conditions. Mycopathology 8(2), 77-80.

Singh, A.K., Mir, M.S., 2005. Genetic variability, heritability and genetic advance in pea (Pisum sativum L.) under cold arid region of Ladakh. Environment and Ecology 23S (Special 3), 445-449.

Singh, I., Singh, P., Sandhu, J.S., 2007. Genetic divergence and association studies in field pea (Pisum sativum L.). Crop Improvement 34(2), 179-182.

Singh, N., Dhillon, G.S., 2006. Genetic variability for pod yield and its contributing traits in garden pea (Pisum sativum L. var. hortense). Haryana Journal of Horticultural Sciences 33(3/4), 300-301. 